\title{
Estimating the Time after Death on the Basis of Corneal Opacity
}

Wataru Kawashima*, Katsuhiko Hatake, Risa Kudo, Mari Nakanishi, Shigehiro Tamaki, Shogo Kasuda, Katsuya Yuui and Akiko Ishitani

Department of Legal Medicine, Nara Medical University School of Medicine, 840 Shijo-cho, Kashihara, Nara 634-8521, Japan

\begin{abstract}
Estimation of the time after death (TAD) is an important aspect of forensic science. The cornea becomes increasingly opaque with an increase in TAD and corneal opacity is used for TAD estimation. However, previous methods are subjective, and there is a risk of human error. To establish an objective method, we propose a new method for TAD estimation.

We applied RGB image analysis to the corneal color of cadavers. We then examined if there was a correlation between these color parameters and actual TAD, age, position at death, and environmental temperature. We found that corneal opacity was affected only by TAD.

The method described here is objective and easy to use, and TAD can be estimated within a very short period of time, making this method particularly useful. To further increase the accuracy of TAD estimation, other quantifiable parameters could also be evaluated.
\end{abstract}

Keywords: Forensic science; Corneal opacity; Computer-assisted image analysis; Time after death; Autopsy; Cadaver

\section{Introduction}

During an autopsy, accurate estimation of the time after death (TAD) is very important. The association between corneal transparency and TAD was established in 1965 [1], and it is now generally accepted in the forensic community that the cornea becomes increasingly opaque with a longer TAD. Therefore, corneal opacity is an accepted means for estimating TAD.

Although the use of corneal opacity in estimating TAD has been established, there are limitations in its application. Owing to the use of macroscopic evaluation, the degree of opacity is classified only as mild, moderate, or high. In addition, the evaluation criteria used to determine the classification rely on the examiner's judgment; therefore, different examiners can reach different conclusions for the same cadaver.

A previously used method of TAD estimation based on corneal opacity is the gray scale method [2], which estimates TAD through comparison of the degree of corneal opacity to the gray scale, but this method also relies on the examiner's judgment. Other, objective means of TAD estimation are also available, including the measurement of laser light transmittance [3,4] and corneal thickness [5], calculation of the number of dead endothelial cells in the cornea [6,7], and estimation of the state of DNA degradation in the corneal cells [8]. However, most of these methods require removal of the eyeball, which affects the appearance of the cadaver. They also require specialized procedures and equipment as well as a considerable amount of time for TAD estimation.

Image analysis is another option for TAD estimation. Zhou et al. [9] and Liu et al. [10] reported the use of image analysis as a TAD estimation method in mice and rabbits. They calculated the degree of corneal opacity on the image according to the TAD and found a correlation between the degree of opacity and TAD; the degree of corneal opacity increased with increasing TAD. However, this technique has not been used for human corneas.

In order to use image analysis for the evaluation of corneal opacity in humans, we devised a simple and objective method for photographing the corneal color of cadavers during an autopsy. Following the use of this newly developed method, we evaluated the relationship between corneal opacity and TAD as well as the influence of several factors that may affect the degree of corneal opacity. Finally, we proposed an estimation method for TAD by evaluating the degree of corneal opacity.

\section{Materials and Methods}

The study protocol was approved by the ethics committees of the Nara Medical University.

The corneal images were collected during autopsies that were performed in the Department of Legal Medicine, Nara Medical University, between April 2011 and December 2012. Cadavers ( $n=174$ ) within $48 \mathrm{~h}$ after death and whose age, gender, and time of death were known or could be identified within a specific time range were used. And none had been stored in the freezer till $48 \mathrm{~h}$ after death. The cadavers with conditions that could affect corneal opacity, such as fire damage, a high level of decomposition, and considerable damage of the craniocervical region, were excluded from the present study.

During the autopsy of each cadaver, photographs of both eyes were taken using a digital camera (D3000; Canon, Japan). We positioned the camera just above the cadaver, and the light source placed slightly left of and above it in order to avoid the reflection of light seen on the cornea when taking photograph. To compare the degree of opacity under different lighting, 54 of the 174 cadavers' corneas were photographed under light from light emitting diodes (LEDs) as well as room light (Figure la and 1b). The time from the known time of death to the time that the photograph was taken was defined as the actual TAD.

*Corresponding author: Wataru Kawashima, Department of Legal Medicine Nara Medical University School of Medicine, 840 Shijo-cho, Kashihara, Nara 634-8521, Japan, Tel: +81-744-29-8843; Fax: +81744-29-1116; E-mail: watack14@naramed-u.ac.jp

Received January 07, 2015; Accepted January 25, 2015; Published January 30 2015

Citation: Kawashima W, Hatake K, Kudo R, Nakanishi M, Tamaki S, et al. (2015) Estimating the Time after Death on the Basis of Corneal Opacity. J Forensic Res 6 : 269. doi:10.4172/2157-7145.1000269

Copyright: (c) 2015 Kawashima W, et al. This is an open-access article distributed under the terms of the Creative Commons Attribution License, which permits unrestricted use, distribution, and reproduction in any medium, provided the original author and source are credited. 

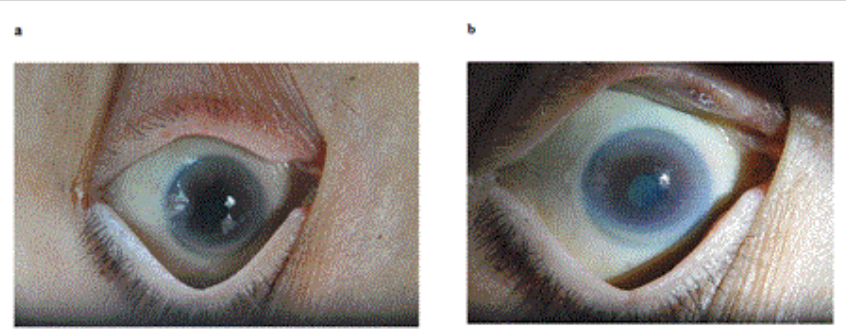

Figure 1: (a and b) Images of cadaveric corneas under room light (a) and LED (b) for the analysis of corneal opacity LED: Light Emitting Diode.

\section{Corneal Color Diagnosis}

From the photographic images, corneal color was defined using RGB shading in an open-source software program (Image J, http:// imagej.nih.gov/ij/index.html). In this program, every color is defined as combinations of shades of red (R), blue (B), and green $(G)$, from black $(R, B, G=0,0,0)$ to white $(R, B, G=255,255,255)$. We chose 5 points of the corneal region (center, top, bottom, right side, left side) for analysis, and the average values for each cornea were used in subsequent analyses (Figure 2).

\section{Statistical Analysis}

All data were analyzed using SPSS v19.0 (IBM Corp, Armonk, NY). Age is expressed as mean (SD) values. Statistical significance was set at a two-tailed $\mathrm{P}$ value of $<0.05$.

Pearson correlation coefficient analyses were conducted to determine relationships between TAD and corneal color (defined as RGB shading) under both room light and LEDs. The actual TAD values were grouped according to 10 -h intervals $(0-9,10-19,20-29,30-39$, and $40-48 \mathrm{~h}$ ). These groups were used to conduct student t-tests between the results obtained in room light and those obtained using LEDs, to investigate the differences in the degree of corneal opacity in both lighting sources.

We classified the cadavers into 6 age groups $(<40,40-49,50-59,60$ $69,70-79$, and $\geq 80$ years), 5 positions at death (dorsal, prone, lateral, hanging, and sitting), and 3 environmental temperature groups $\left(<10^{\circ} \mathrm{C}\right.$, $10-20^{\circ} \mathrm{C}$, and $\geq 30^{\circ} \mathrm{C}$ ) to investigate the effects of age, position at death, and environmental temperature on corneal opacity; these effects were analyzed using an ANCOVA. The Pearson correlation coefficient was also determined for TAD and corneal color in each group.

Multiple regression model analysis was conducted to determine the effect of the variables that influenced corneal opacity on TAD estimation; from this, we proposed equations for estimating TAD using RGB shading under room light and LEDs. Multi-collinearity was assessed using the variance inflation factor (VIF) for each parameter prior to the regression analysis.

We performed data splitting for cross-validation of the model. The calculated TAD estimates were then compared to the recorded actual TAD of the cadavers; the calculated differences between the estimated and actual TAD are referred to as the mean error.

\section{Results}

Eyes from 104 male and 70 female cadavers (average age, 63.9 [18.1] years and 68.9 [21.9] years, respectively) were evaluated in this study. The average TAD was 20.0 (9.3) h (range: $3-48 \mathrm{~h}$ ).
There were positive correlations between corneal color, as estimated from RGB shading, and actual TAD for both light sources (Table 1, Figure 3a and 3b), and the degree of corneal opacity increased with increasing TAD. The t-test results indicated a significantly larger mean RGB value in the presence of LEDs than that in room light over the entire time period and in each TAD 10-h category, except for the category of 40-48 h (Figure 4).

Significant positive correlations were also observed between TAD and corneal color, defined using RGB shading, in each group classified by age, position at death, and environmental temperature (Table 2). The correlation between TAD and RGB shading increased with increasing age; however, the correlations for environmental temperature and position at death were similar across all categories. The results of the ANCOVA revealed no relationship between corneal opacity and each factor (age, position at death, and environmental temperature).

Based on these results, proposed equations for estimating TAD using corneal color analysis are provided in Table 3. No parameter had a VIF $\geq 10$, allowing for the inclusion of all parameters in the regression model. Although age was not a significant factor in the ANCOVA results, it was included in the equations because of the correlations between increasing age and greater corneal opacity. Using these equations, the differences between the estimated and actual TAD, referred to as the mean error, were found to be $6.3(4.1) \mathrm{h}$ for the room light condition and 9.7 (7.6) $\mathrm{h}$ for the LED condition (Table 4). The mean error of the estimation in the room light condition was significantly less than that of the estimation in the LED condition.

In the room light condition, the mean error was within $5 \mathrm{~h}$ of the actual TAD in $50 \%$ of the estimated TAD values (Figure 5). Further assessment of the mean errors showed that 31 cases had mean errors $>10 \mathrm{~h}$, and these 31 cadavers were all aged over 60 years.

When comparing the TAD 10-h categories, the mean errors of the categories $>10 \mathrm{~h}$ and $<30 \mathrm{~h}$ were each significantly lower than those of the other TAD categories (Figure 6).

\section{Discussion}

The results of this study demonstrate the usefulness of computerbased image analysis in the estimation of TAD during an autopsy. The

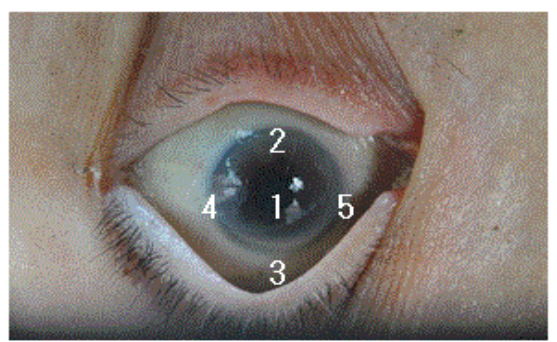

Figure 2: Five points of the corneal region used for the calculations of the average RGB shading to determine corneal opacity.

\begin{tabular}{|c|c|c|c|}
\hline & \multicolumn{3}{|c|}{ Correlation coefficient (r) } \\
\hline & Room light & LED & P value \\
\hline TAD \& R & 0.60 & 0.35 & $<0.01$ \\
\hline TAD \& G & 0.60 & 0.40 & $<0.01$ \\
\hline TAD \& B & 0.56 & 0.42 & $<0.01$ \\
\hline
\end{tabular}

Table 1: Relationships between TAD and corneal color (defined as RGB shading) under 2 light conditions-room light and LED. R: Red; B: Blue; G: Green; TAD: Time after Death; LED: Light Emitting Diode. 
a
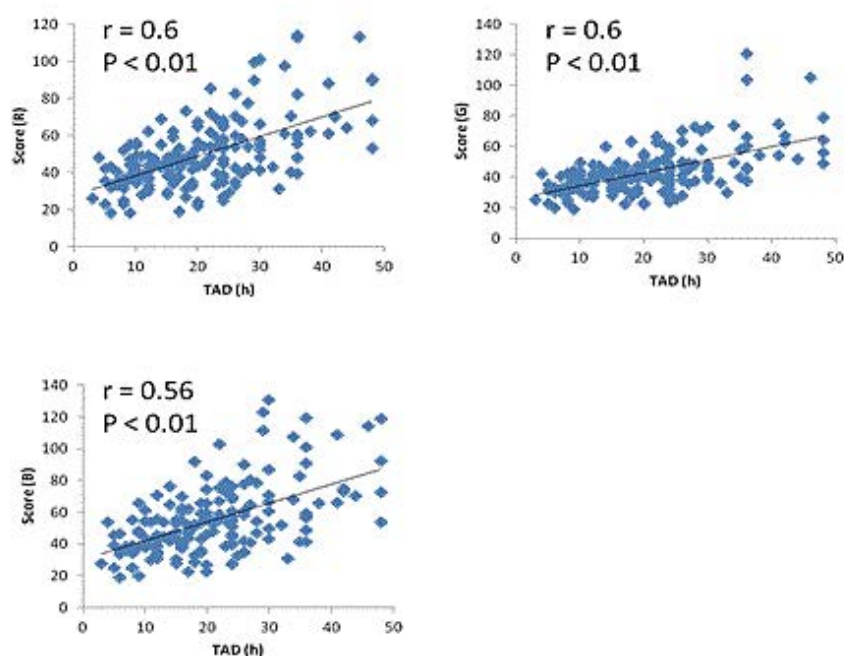

b
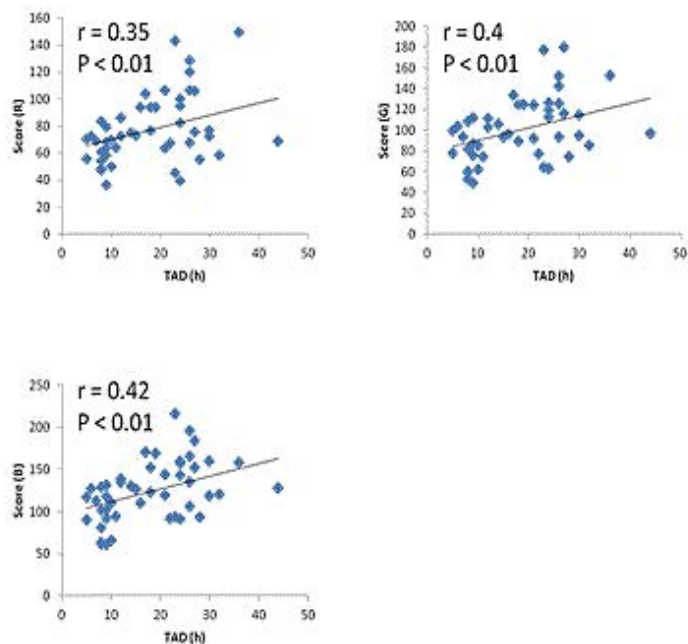

Figure 3: $(a$ and $b)$ Correlations between time after death (TAD) and corneal color (defined as RGB shading) in the room light condition (a) and LED condition (b) R: Red; B: Blue; G: Green; LED: Light Emitting Diode.

$0-9 \mathrm{~h}$

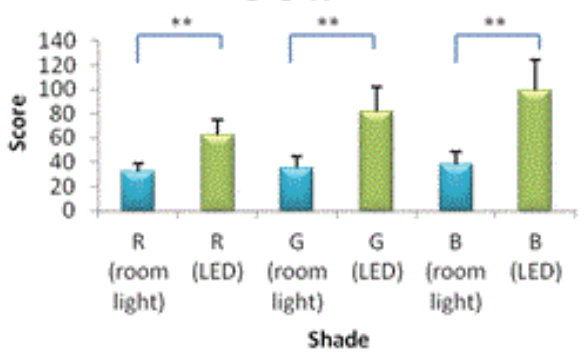

20-29 h

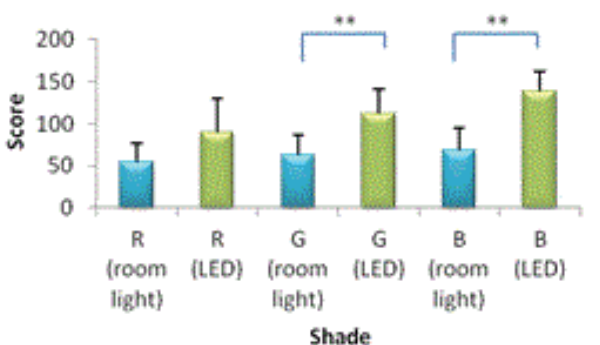

40-48 h

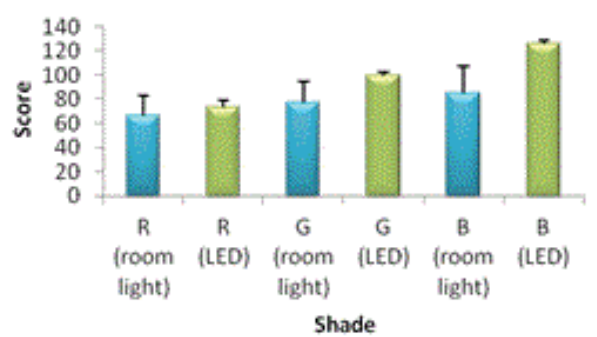

$10-19 \mathrm{~h}$

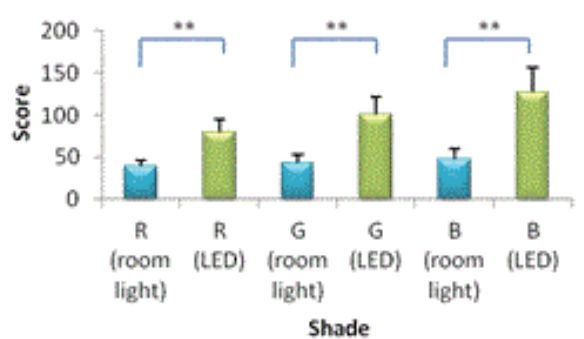

30-39 h

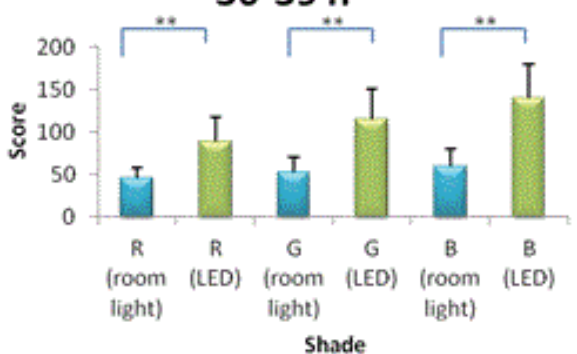

Total

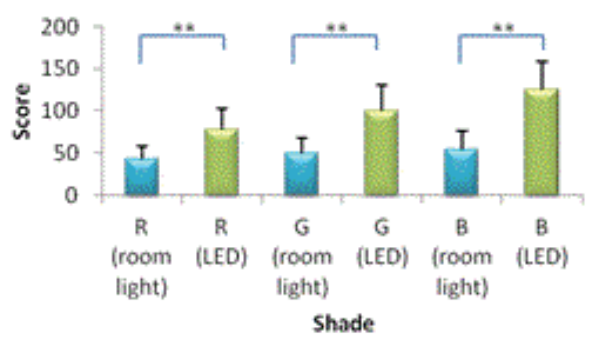

Figure 4: Comparison of the mean RGB value under room light and LED over the entire time period and in each $10-\mathrm{h}$ category. ${ }^{* *} \mathrm{P}<0.01$, comparison between room light and LED. R: Red; B: Blue; G: Green; LED: Light Emitting Diode. 
Page 4 of 5

\begin{tabular}{|c|c|c|c|c|c|c|}
\hline & TAD\&R & $P$ value & TAD\&G & $P$ value & TAD\&B & $P$ value \\
\hline \multicolumn{7}{|c|}{ Age category (years) } \\
\hline$<40$ & 0.43 & $<0.01$ & 0.41 & $<0.01$ & 0.36 & $<0.01$ \\
\hline $40-49$ & 0.44 & $<0.01$ & 0.44 & $<0.01$ & 0.44 & $<0.01$ \\
\hline $50-59$ & 0.62 & $<0.01$ & 0.54 & $<0.01$ & 0.47 & $<0.01$ \\
\hline $60-69$ & 0.61 & $<0.01$ & 0.57 & $<0.01$ & 0.52 & $<0.01$ \\
\hline $70-79$ & 0.69 & $<0.01$ & 0.64 & $<0.01$ & 0.58 & $<0.01$ \\
\hline$\geq 80$ & 0.65 & $<0.01$ & 0.73 & $<0.01$ & 0.75 & $<0.01$ \\
\hline \multicolumn{7}{|c|}{ Position at death } \\
\hline Dorsal & 0.61 & $<0.01$ & 0.61 & $<0.01$ & 0.56 & $<0.01$ \\
\hline Prone & 0.61 & $<0.01$ & 0.57 & $<0.01$ & 0.52 & $<0.01$ \\
\hline Lateral & 0.69 & $<0.01$ & 0.66 & $<0.01$ & 0.66 & $<0.01$ \\
\hline Hanging & 0.5 & $<0.01$ & 0.51 & $<0.01$ & 0.52 & $<0.01$ \\
\hline Sitting & 0.65 & $<0.01$ & 0.69 & $<0.01$ & 0.7 & $<0.01$ \\
\hline \multicolumn{7}{|c|}{ Environmental temperature $\left({ }^{\circ} \mathrm{C}\right)$} \\
\hline$<10$ & 0.61 & $<0.01$ & 0.61 & $<0.01$ & 0.56 & $<0.01$ \\
\hline $10-20$ & 0.61 & $<0.01$ & 0.57 & $<0.01$ & 0.52 & $<0.01$ \\
\hline$\geq 30$ & 0.69 & $<0.01$ & 0.66 & $<0.01$ & 0.66 & $<0.01$ \\
\hline
\end{tabular}

Table 2: The correlation coefficients for the relationships between TAD and corneal color (defined as RGB shading) displayed as groups by age, position at death, and environmental temperature of the cadaver. R: Red; B: Blue; G: Green; TAD: Time after Death.

\begin{tabular}{|c|c|c|}
\hline & Room light & LED \\
\hline & TAD $=-0.06$ age $+0.2 R-0.01 G+0.13 B+29.5$ & TAD $=-0.17$ age $-0.005 R-0.33 G+0.6 B+69.9$ \\
\hline$R 2$ & 0.69 & 0.81 \\
\hline Adjusted R2 & 0.47 & 0.66 \\
\hline
\end{tabular}

Table 3: Equations for the estimation of TAD using photographic corneal color evaluation in shades of R, B, and G under 2 different light conditions (room light and LED). R: Red; B: Blue; G: Green; TAD: Time after Death; R: multiple correlation coefficient; R2: coefficient of determination; LED: Light Emitting Diode.

\begin{tabular}{|c|c|c|c|c|c|c|}
\hline \multirow[b]{2}{*}{ TAD (h) } & \multicolumn{3}{|c|}{ Room light condition } & \multicolumn{3}{|c|}{ LED condition } \\
\hline & Mean error (h) & S.D. & $\mathbf{N}$ & Mean error (h) & S.D. & $\mathbf{N}$ \\
\hline $0-9$ & 8.9 & 3.5 & 27 & 9.9 & 7.6 & 14 \\
\hline $10-19$ & 4.5 & 3.1 & 52 & 7 & 6.5 & 12 \\
\hline $20-29$ & 4.5 & 2.7 & 51 & 11.2 & 7.8 & 16 \\
\hline $30-39$ & 10.2 & 5 & 27 & 10.1 & 8.6 & 6 \\
\hline $40-48$ & 10.2 & 5.5 & 17 & 11.1 & 14.8 & 6 \\
\hline All & 6.3 & 4.1 & 174 & 9.7 & 7.6 & 54 \\
\hline
\end{tabular}

Table 4: The mean error of the TAD equation, compared to actual TAD, when using the corneal color analysis from photographs taken in 2 different light conditions (room light and LED). S.D., standard deviation; TAD: Time after Death; LED: Light Emitting Diode.

positive relationships that existed between RGB shading in the cornea and actual TAD indicated that the cornea became opaque (higher RGB score) with increasing TAD. These results support those reported previously by Aoki [1].

Computer-based image analysis offers the benefit of digitization, which confers many advantages such as stability, reliability, noninvasiveness, reproducibility, and the elimination of human error. In this study, we adapted the image analysis methodology for humans by using the RGB score to calculate the degree of opacity. Because the RGB score can indicate the color of the cornea through the choice of specific points on the cornea, it improves the objectivity, simplicity, and speed of the analysis. Moreover, the RGB score reflects an objective evaluation of opacity, further eliminating the risk of human error while, at the same time, providing a segmentalized numerical score comprised of each color (R, G, B) on a scale of 0 to 255 .

Despite the superiority of image analysis over other methods for estimating TAD, several factors affecting the quality of the photograph and the condition of the cornea should be considered. Photographs in previous studies were taken under room light; the effect of the light source on corneal opacity was not taken into consideration. In this study, LEDs resulted in a higher degree of corneal opacity than room light for the TAD 10-h categories $<40 \mathrm{~h}$. The light produced by LEDs may be brighter than room light, resulting in larger values for the RGB score and a more opaque corneal color. Moreover, the slight change in corneal color with TAD was not apparent under LEDs, as indicated by the smaller correlation coefficient with LEDs than that with room light. In addition, the mean error of the equation developed with room light was significantly smaller than that of the LED equation, leading us to believe that room light is more acceptable for this method of TAD estimation.

Corneal opacity can also occur due to transpiration of fluid and degeneration of endothelial cells and may therefore be accelerated in high temperatures. Corneal opacity may also be affected by postmortem hypostasis that occurs when the person is in a prone position at the time of death. Age is another factor to consider, as it is generally accepted that corneal opacity increases with age due to a decrease in the number of corneal endothelial cells, which results in an increase 


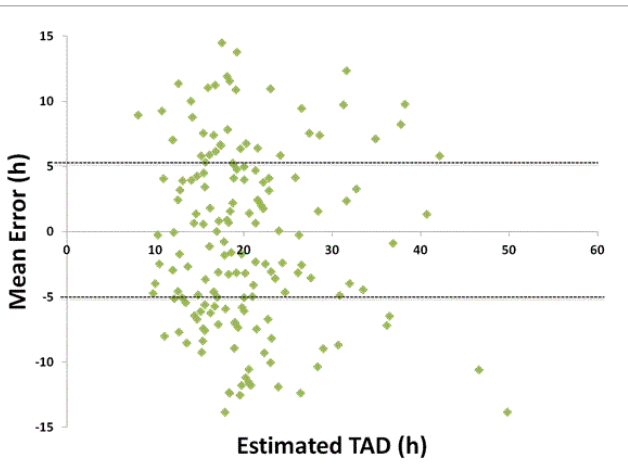

Figure 5: The mean error of the TAD equation, compared to the actual TAD, when using the corneal color analysis from photographs taken in room light. TAD: Time After Death.

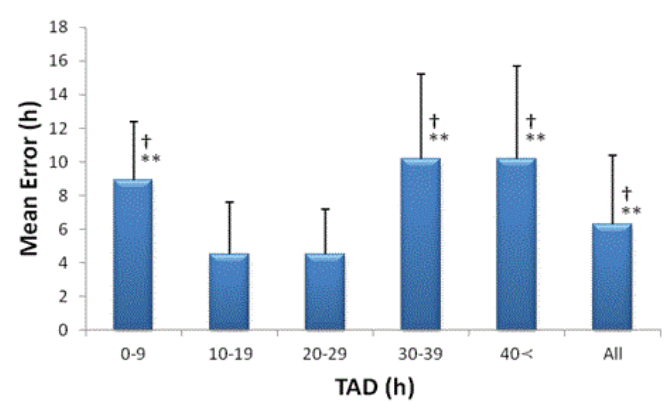

Figure 6: Comparison of the mean error in the estimation of TAD, compared to the actual TAD in each of the 10-h TAD intervals, using corneal color analysis in photographs taken in room light. $† \mathrm{P}<0.01$, compared to $10-19 \mathrm{~h},{ }^{* *} \mathrm{P}<0.01$, compared to 20-29 h, TAD: Time after Death.

in the thickness of the cornea owing to inflow of aqueous humor to the cornea. Therefore, we investigated the effect of these factors (age, environmental temperature, and position at death) on corneal opacity using ANCOVA, and the results indicated that the factors have no effect on corneal opacity. Therefore, it may not be necessary to consider their effect within $48 \mathrm{~h}$ of death.

In this study, we also developed equations to estimate TAD from RGB image analysis in room light. Although the total mean error of the room light equation was considered to be small $(6.3[4.1] \mathrm{h})$, the mean errors at the shorter $(<9 \mathrm{~h})$ and longer $(>30 \mathrm{~h})$ TAD times were significantly larger. The shorter time period would result in very little change in corneal opacity, while the longer time period would result in a large change in corneal opacity. The cadavers with mean errors $>10 \mathrm{~h}$ were all aged over 60 years, indicating that care should be taken when using this method in older people.

The results of our image analysis, an objective method, confirm that corneal color increases in opacity with longer TADs. Autopsies performed within 10-30 h of death can confidently use this method for accurate estimation of TAD. However, with shorter and longer TAD, the accuracy may diminish. Despite these limitations, this estimation method is the first to support the use of image analysis to determine corneal color. Thus, it may be a useful alternative. As the use of LEDs becomes more common, it will be necessary to develop a more accurate method for TAD estimation under LEDs.

\section{References}

1. Aoki T (1965) Studies on the estimation of time after death. Jikeikai Med J 1:3-18.

2. Furukawa M, Funao T, Nagasaki $Y$ (1985) A new estimation of lapse of time since death by corneal turbidity. Kitasato Med 15: 364-367.

3. Tsunenari S, Watanabe S, Takahama K, Kanda M (1971) A method to determine the corneal turbidity by application of laser. Nihon Hoigaku Zasshi 25: 373-375

4. Tsunenari S, Oho S, Sasaki S, Muto H, Kanda M (1974) The development of the laser apparatus to determine corneal turbidity-model. Jpn J Leg Med 28: 281-285.

5. Meng YZ, Fu CJ, Wang C (2001) An experimental pathological study of the relationship between the corneal thickness and the time of death. Hubei Yikedaxue Xuebao 22: 35-36.

6. Song G, Shi JH (2001) Study of corneal endothelium cell and its application in forensic medicine. Forensic Sci Technol 1: 36-37.

7. Song GL, Shi JH, Qiao H, et al. (1995) Estimation time of death by determining the endothelial cell living rate from corpse cornea. Chin J Forensic Med 10: 90-93.

8. Chen X, Yi S, Liu L (2007) Image analysis for degradation of DNA in retinal nuclei of rat after death. J Huazhong Univ Sci Technol Med Sci 27: 24-26.

9. Zhou L, Liu Y, Liu L, Zhou L, Liang M, et al. (2010) Image analysis on corneal opacity: A novel method to estimate postmortem interval in rabbits. J Huazhong Univ Sci Technol Med Sci 30: 235-239.

10. Liu F, Zhu S, Fu Y, Fan F, Wang T, et al. (2008) Image analysis of the relationship between changes of cornea and postmortem interval. In: Ho T-B, Zhou Z-H (eds.) PRICAI: Trends in artificial intelligence. Springer, Berlin 998-1003. 